Marquette University

e-Publications@Marquette

3-2018

\title{
Estimating HIV Medication Adherence and Persistence: Two Instruments for Clinical and Research Use
}

David A. Wohl

University of North Carolina - Chapel Hill

A. T. Panter

University of North Carolina - Chapel Hill

Christine Kirby

University of North Carolina - Chapel Hill

Brooke E. Magnus

Marquette University, brooke.magnus@bc.edu

Michael G. Hudgens

University of North Carolina - Chapel Hill

See next page for additional authors

Follow this and additional works at: https://epublications.marquette.edu/psych_fac

Part of the Psychology Commons

\section{Recommended Citation}

Wohl, David A.; Panter, A. T.; Kirby, Christine; Magnus, Brooke E.; Hudgens, Michael G.; Allmon, Andrew G.; and Mollan, Katie R., "Estimating HIV Medication Adherence and Persistence: Two Instruments for Clinical and Research Use" (2018). Psychology Faculty Research and Publications. 294.

https://epublications.marquette.edu/psych_fac/294 


\section{Authors}

David A. Wohl, A. T. Panter, Christine Kirby, Brooke E. Magnus, Michael G. Hudgens, Andrew G. Allmon, and Katie R. Mollan 
Marquette University

e-Publications@Marquette

\section{Psychology Faculty Research and Publications/College of Arts and Sciences}

This paper is NOT THE PUBLISHED VERSION; but the author's final, peer-reviewed manuscript. The published version may be accessed by following the link in th citation below.

AIDS \& Behavior, Vol. 22, No. 3 (March 2018): 948-960. DOI. This article is (C) Springer and permission has been granted for this version to appear in e-Publications@Marquette. Springer does not grant permission for this article to be further copied/distributed or hosted elsewhere without the express permission from Springer.

\section{Estimating HIV Medication Adherence and Persistence: Two Instruments for Clinical and Research Use}

David A. Wohl

Division of Infectious Diseases, The University of North Carolina, Chapel Hill, NC

A. T. Panter

Department of Psychology and Neuroscience, The University of North Carolina, Chapel Hill, NC

Christine Kirby

Division of Infectious Diseases, The University of North Carolina, Chapel Hill, NC

Brooke E. Magnus

Psychology Department, Marquette University, Milwaukee, WI

Michael G. Hudgens

Department of Biostatistics, The University of North Carolina, Chapel Hill, NC

Andrew G. Allmon

Department of Biostatistics, The University of North Carolina, Chapel Hill, NC

Katie R. Mollan

The University of North Carolina Center for AIDS Research, Chapel Hill, NC 


\section{Abstract}

Antiretroviral therapy (ART) requires lifelong daily oral therapy. While patient characteristics associated with suboptimal ART adherence and persistence have been described in cohorts of HIV-infected persons, these factors are poor predictors of individual medication taking behaviors. We aimed to create and test instruments for the estimation of future ART adherence and persistence for clinical and research applications. Following formative work, a battery of 148 items broadly related to HIV infection and treatment was developed and administered to $181 \mathrm{HIV}$-infected patients. ART adherence and persistence were assessed using electronic monitoring for 3 months. Perceived confidence in medication taking and self-reported barriers to adherence were strongest in predicting non-adherence over time. Barriers to adherence (e.g., affordability, scheduling) were the strongest predictors of non-adherence, as well as 3- and 7-day non-persistence. A ten-item battery for prediction of these outcomes (www.med.unc.edu/ncaidstraining/adherence/for-providers) and a 30-item battery reflective of underlying psychological constructs can help identify and study individuals at risk for suboptimal ART adherence and persistence.

\section{Keywords}

Adherence HIV Item response theory Measurement Prediction

\section{Introduction}

Advances in antiretroviral therapy (ART) have significantly improved the prognosis and quality of life of persons living with HIV infection, and developments in therapeutics, including fixed-dose combination formulations and single tablet regimens, have simplified ART without compromising efficacy. However, the success of ART continues to hinge on adequate adherence and persistence to these medications, which, at present, require lifelong daily dosing.

Suboptimal adherence to treatment and care remains a challenge for many patients, even in the era of modern $A R T$, and can lead to virologic failure, drug resistance, and transmission of the virus [[1] -[4] ]. Studies conducted in disparate HIV-infected populations reveal that medication taking behaviors can vary considerably, are complex, and influenced by personal, institutional, and structural factors [[1] , [2] , [5] - [9] ]. Mental health challenges, substance abuse, poor social support, unstable housing, denial, stigmatization, regimen complexity, and cost have each been associated with sub-optimal ART adherence [[1] , [2] ]. These correlates of ART adherence, derived from cohort studies, are useful when examining populations, but are imperfect predictors of ART adherence at the individual level. Therefore, clinicians are frequently left to rely on judgment and intuition when assessing the likelihood of a patient taking their medication regularly-resulting in inaccurate predictions of patient adherence [[10] ].

Using two scale development approaches, we aimed to create and test an instrument that would allow clinicians and researchers to estimate future adherence and persistence to ART. We recruited a diverse sample of HIVinfected outpatients receiving care and recruited patients in clinics located in North Carolina, a state with the eighth highest HIV prevalence rate in the United States. We reasoned that this new instrument could be used to identify patients/participants at risk for missing ART doses and, therefore, could also identify candidates for interventions to support adherence and prevent treatment failure. 


\section{Methods}

\section{Survey Development}

The survey development process included three phases: Formative, Instrument Development, and Instrument Piloting and Testing. The University of North Carolina at Chapel Hill (UNC-Chapel Hill) Biomedical Institutional Review Board approved all phases.

Formative Phase

Individual semi-structured interviews were conducted with 21 stakeholders in North Carolina, including staff (e.g., physicians, nurses, social workers) at major HIV clinics and AIDS service organizations, and state health officials involved in HIV-related policy-making, to assess their perspectives regarding challenges to ART adherence. Subsequently, six focus groups were conducted among 56 HIV-infected patients who had been prescribed ART, and were at least 18 years of age. Two focus groups were organized in each of three geographically distinct areas of North Carolina, including a major metropolitan center, a smaller city, and a rural county. One focus group was conducted in Spanish. Participants were recruited via flyers placed in HIV clinics and community service organizations located in these areas. The focus group guide was informed by the stakeholder responses and the published literature on ART adherence challenges and was designed to explore attitudes regarding ART among those living with the virus. Findings from the Formative Phase were previously reported [[11], [12] ].

\section{Instrument Development Phase}

Focus group data were coded and analyzed to identify themes related to the experience of taking ART. The identified themes were used to generate survey items-statements that could be read or spoken to elicit a response. In total, 94 Likert-type items with a five-point agreement scale ( 1 = strongly disagree; 5 = strongly agree) and 11 contingency questions, which included an additional response option of does not apply, were combined to create a final battery of items. To gain a better understanding of these items and how they might be understood by our sample, cognitive interviews were conducted with $18 \mathrm{HIV}$-positive patients receiving care at the University of North Carolina (UNC) Infectious Diseases Clinic. The HIV Treatment Adherence Self-Efficacy Scale (HIV-ASES) [[13] ] and a modified version of the AIDS Clinical Trials Group Adherence Barriers Questionnaire (ACTG-ABQ) (dichotomous yes/no responses were used instead of the original four-point Likert scale) [[14] ] were also administered. At the end of this phase of instrument development, 148 items were included in a question battery for use in the Instrument Piloting and Testing Phase.

Instrument Piloting and Testing Phase

The question battery was piloted with 60 HIV-infected patients receiving care at one of four HIV treatment centers: the UNC-Chapel Hill Infectious Diseases Clinic, the Wake County Early Intervention Clinic in Raleigh, the Wake Forest Medical Center Infectious Diseases Clinic in Winston-Salem, and the Regional Center for Infectious Diseases in Greensboro. The inclusion criteria were as follows: age 18 years or older, English speaking, able to provide informed consent, receiving ART, not using a pill box, and not residing in an institutional setting where medications are directly administered. The derived instrument is intended to be used in patients with unknown levels of adherence. There was no adherence inclusion criterion as a broad spectrum of pill-taking behaviors was sought to allow for determination of item performance and discrimination. Responses from patients with high levels of adherence and persistence were necessary to identify response patterns that are predictive of suboptimal adherence. As with prior stages of the instrument development process, participants were recruited using flyers placed in HIV clinics and community service organizations. Interested patients called a research number and were screened by a research associate not associated with the recruitment clinics. 
Following the survey piloting, minor wording changes were made to the survey battery. An additional 121 patients meeting the same inclusion criteria for the pilot were recruited using identical procedures at the same four HIV treatment centers to assess the predictive performance of the items in relation to ART adherence and persistence. As described in detail below, participants were followed for 3 months during which ART adherence was assessed. The results from the combined samples from the piloting and testing phases $(60+121)$ are reported in this manuscript.

\section{Study Procedures}

\section{Assessments: Instrument Piloting and Testing Phase}

For this study phase, participants were screened by research associates who were not associated with the clinics from which participants were recruited. Those confirmed to be eligible completed a baseline study visit during which the battery of items was administered using a tablet computer that the participant used to indicate their responses. At the visit, an electronic medication monitoring cap (MEMs, Aardex Inc) was placed on one HIV medication bottle. The MEMs cap was placed preferentially on the antiretroviral that the patient self-reported as most demanding for them; typically this was the medication that required the greatest number of doses per day, number of pills per day, or largest pill.

After the baseline visit, participants were seen at a mutually agreed upon location where the study visit could be conducted safely and confidentially at monthly intervals for 3 months. ART adherence and persistence were assessed by MEMs in the Instrument Piloting and Test Phase. The visual analog scale was used to assess baseline ART adherence among those entering the study on HIV therapy [[15] ].

Demographic characteristics, self-reported health status, HIV and medical history, ART taking practices (i.e., pill box use, pocketed doses), motivation to adhere to ART, substance use, and depression were obtained at baseline by a trained project staff member via computer assisted patient interviewing (CAPI). At each follow-up visit, changes in health and medication were recorded.

\section{Data Analytic Approach}

\section{Quantifying Adherence and Persistence}

To assess a participant's non-adherence, a ratio was calculated as the total number of non-adherent days divided by the number of days the participant was monitored by MEMs cap. If the number of bottle openings matched or exceeded the expected dose frequency, then the daily adherence was considered a success. A participant with a scheduled, twice daily dose and only one bottle opening for the day was considered nonadherent for the day. The adherence estimates for the first and last days the MEMs cap use were excluded as the adherence data on these days would be partial. The adherence ratio (0-100\%) was analyzed and suboptimal adherence was also defined using 2 cutoffs, $>20$ and $>30 \%$ non-adherence. There is no consensus regarding the threshold of optimal ART adherence. Thus we chose to examine non-adherence rates of 20 and $30 \%$ and assume that those who take at least $80 \%$ of their ART would be at low risk of treatment failure while those who take less than $70 \%$ of their HIV medication would have a substantially greater likelihood of failure to achieve or maintain viral suppression.

ART non-persistence was examined using a 3- and 7-day threshold for permissible gaps. Non-persistence was quantified as the number of times a participant failed to open their bottle for three or more consecutive days (i.e., three zeros in a row); this measure was also quantified using seven or more consecutive days of missed bottle openings. We refer to these as "3-" and "7-day" non-persistence, respectively.

MEMs data were analyzed using two approaches: (1) a "conservative" analysis in which no adjustment to the recorded MEMs data were made; and (2) an "adjudicated" analysis in which MEMs data were censored based 
on participant self-report of non-use of the MEMs cap. The most common reasons for data censoring in the adjudicated approach were intermittent use of a pillbox and pocketing of doses (e.g., removing the evening dose of a twice a day medication when taking the morning dose). Conservative results were comparable to adjudicated results; unless otherwise stated, the adjudicated results are shown.

\section{Psychometric Analyses to Identify Reliable Item Subscales Using Item Response Theory}

To evaluate which items included in the Instrument Piloting and Test Phase comprise psychometrically sound scales, we examined the factor structure of each scale as it was blocked and presented to respondents on the baseline survey. We used the software IRTPRO 2.0 [[16] ] to compute local dependence (LD) statistics for each item block from the baseline survey. Large LD values for item pairs suggested that those particular items share specific variance above and beyond the other items in the survey block. Clusters of items exhibiting large LD statistics suggested possible underlying factors; we formed subscales based on these suggested factors. We then calibrated the items and scored respondents, such that each respondent had a single item response theory (IRT) scale score for each subscale. IRT scale scores represent responses to categorical and ordinal items that have been transformed into a continuous scale based on each specific response pattern.

Using item-level responses from baseline assessments, we identified reliable subscales based on attitudes, selfreported adherence behavior, and beliefs. To determine which items within a subscale had the most desirable psychometric properties, we examined trace lines and item discrimination parameters. Items with larger discrimination parameters are better able to differentiate between people across levels of the underlying factor; therefore, those items with the greatest discriminating power were considered the strongest candidates for each subscale.

Statistical Analyses to Predict Medication Adherence from Individual Items

To identify candidate scale items that could be strong predictors of non-adherence and non-persistence, we also fit penalized regression models using the multivariable lasso selection procedure [[17] ]. The lasso approach is useful when there are many items to consider in a prediction problem because it avoids model over-fitting. Models were cross-validated and used to select the lasso penalization tuning parameter for each model.

Two types of predictor variables were used in these analyses, separately: (1) the 148 individual survey items; and (2) the 12 IRT reliable subscales identified in the prior IRT data analytic step. The 11 contingency survey items were recoded into three categories prior to analysis (agree, disagree/neutral, does not apply). To predict the non-adherence ratio the count of non-adherent days was modeled using Poisson lasso regression with natural-log transformed number of MEMs monitored days included as the model offset term. Non-persistence was analyzed as a binary outcome ( 0 episodes vs. 1 or more episodes) and modeled using logistic lasso regression. Likewise, binary outcomes of non-adherence $>20$ or $>30 \%$ were modeled with logistic lasso regression. In total, the lasso selection was conducted separately for five endpoints: (1) non-adherence rate; (2) non-adherence >20\%; (3) non-adherence $>30 \%$; (4) 3-day non-persistence; and (5) 7-day non-persistence.

We used multiple imputation with fully conditional specification. Ordinal items were imputed using predictive mean matching, binary items were imputed with logistic regression, and 3-category contingency items were imputed with a generalized logit model. Adherence ratio, 3-day non-persistence, and all fully-observed ordinal survey items were included in the imputation models when feasible. Models including individual survey items used ten imputed datasets and individual predicted values were calculated from the average predicted value over the ten imputed datasets.

We summarized the predictive impact by ranking individual items according to the number of times they were selected by the lasso method across the five endpoints and ten multiple imputed datasets. Individual items that ranked within the top ten were selected for a final prediction model. When lasso-selected items were identified, 
we matched them to the reliable IRT subscales previously created. We conducted Poisson regression (negative binomial regression in the case of statistically significant over-dispersion) and logistic regression to obtain predicted values from combinations of the included IRT subscales and their corresponding items. For nonadherence rate we plotted the "predicted versus observed" rates, and the proportion of predicted values within $\pm 10 \%$ of the observed rate is shown. For each binary outcome, we examined the true positive rate (sensitivity) versus false positive rate (1-specificity) in a receiver-operator characteristic (ROC) curve and the area under the ROC curve (AUROCC).

The study participant-specific relative odds of non-adherence on weekends (Saturday or Sunday) as compared to non-adherence on weekdays was estimated using mixed effects logistic regression with a random intercept for each participant. The "glmnet" package was used to conduct the lasso analyses in R version 3.1.2; other statistical analyses were conducted in SAS 9.3 or 9.4 .

\section{Results}

\section{Participant Characteristics and Disposition}

Of 212 patients approached for enrollment to the Instrument Piloting and Testing Phase, 181 (85\%) consented, entered the study, and completed a baseline survey. Eight participants were excluded from the analysis cohort because there was evidence they used a pill box and not medication bottles on which the MEMs cap was placed $(n=5)$ or did not complete the baseline survey $(n=3)$. The remaining 173 ( $96 \%$ of 181$)$ were considered eligible for analysis. These participants were monitored by MEMs cap for a median $(Q 1, Q 3)$ of $92(86,97)$ days $(10-90$ th percentile: 75-102). A summary of the selected antiretroviral medications for the MEMS cap is provided in Supplemental Table S1. The most common MEMS monitored medications were tenofovir disoproxil fumarate (TDF)/emtricitibine (FTC) (29\%), TDF/FTC/efavirenz (22\%), and TDF/FTC/elvitegravir/cobicistat (12\%).

Table 1 presents the characteristics of the study participants. Most participants were African-American and middle aged; slightly more than a third were women. At study entry, all but two participants were already receiving ART at the time of study entry, and almost $80 \%$ entered the study with an HIV RNA level that was below the limit of assay detection. The median duration of ART therapy was 8 years, and a variety of ART were prescribed, with two thirds of patients reporting being on a once-daily regimen and the remainder reporting being on twice daily regimens. Nearly half of the participants reported a history of depression or bipolar disorder.

\section{Descriptive Findings for ART Adherence and Persistence}

Using the adjudicated MEMs approach (i.e., MEMs data supplemented by respondent self-report), the mean non-adherence rate (non-adherent days/monitored days) was $22 \%$ (SD, 25) over the course of the 3-month study, with a median $(Q 1, Q 3)$ of $13 \%(3,33)$. With the conservative approach (i.e., MEMs data only), the estimated non-adherence rate was similar, with mean (SD) of $25 \%(26)$ of days and median $(Q 1, Q 3)$ of $15 \%$ (3, 37 ) of days. Using the adjudicated approach, $39 \%$ of participants (68/173) had a non-adherence rate $>20 \%$ of days, and $27 \%$ of participants (47/173) had a non-adherence rate $>30 \%$. Patient-specific odds of non-adherence were $30 \%$ higher on the weekends compared to weekdays (estimated odds ratio (OR) $1.30,95 \% \mathrm{Cl}: 1.17-1.44$ ).

Many participants persistently opened their MEMS cap as prescribed, with zero episodes of non-persistence. Nevertheless, at least one episode of non-persistence for 3 days or longer was recorded in $37 \%$ of participants (64/173) during study follow-up, and 17\% (29/173) had at least one episode of non-persistence lasting 7 days or longer. 
Findings from the Psychometric Analyses of Subscales Using Item Response Theory We conducted exploratory factor analyses, followed by assessments of local dependence among item sets, which resulted in 12 subscales from the baseline assessment with moderate to large IRT marginal reliabilities. Appendix Table 4 presents the 12 subscales along with their marginal reliabilities. Descriptive analyses generally showed a negative skew, such that most participants endorsed the upper portions of the scale corresponding to the "positive" set of choices. Participants acknowledged the importance of social support, cited positive reasons for medication (subscale: Positivity), did not let the side effects prevent them from taking the medications (Effects of Medication), and reported trusting their doctors (Trust).

\section{Findings from Predictive Adherence and Persistence Models}

For the adherence predictive models we considered 148 baseline survey items for inclusion in both the IRT subscales and the predictive models of non-adherence and non-persistence. For multivariable analyses of survey items, data were imputed as described in the methods. Of the reliable IRT subscales, HIV-ASES (confidence in medication taking), ACTG-ABQ (barriers to adherence, e.g. busy and forgetful), Difficulty, and Positivity each included one item that ranked in the top ten according to individual item lasso selection; these IRT subscales were used to form prediction models (Table 2). When lasso selection was applied directly to the 12 continuous IRT scores ACTG-ABQ was selected across all five endpoints, Difficulty was selected for four endpoints (not 7-day non-persistence), and Positivity selected for the three adherence endpoints. While HIV-ASES was never selected when fit as an IRT score, the highest ranked individual predictive item was from this subscale, and thus it was retained for the final IRT-based prediction models. Additional IRT scores ever selected by lasso were Mode (7day non-persistence), Convenience (3-day non-persistence), and Trust (3-day non-persistence).

The two candidate surveys arising from this work, Lasso-10 and IRT-30, are presented in Appendix Tables 5 and 6 , respectively, and their internal prediction performance is shown in Table 2. The Lasso-10 included the top ten ranked items using lasso selection without regard to IRT subscales (one item from HIV-ASES, one item from ACTG-ABQ, and eight items novel to this work) with a marginal reliability of.77. The IRT-30 contained 30 items from four IRT subscales and had a marginal reliability of.90. These two potential surveys have four items in common. The ten-item survey takes approximately $5 \mathrm{~min}$ to complete and the 30-item survey takes approximately $10 \mathrm{~min}$ (note the yes/no items were less time consuming than those on a Likert scale). The top ten individual items, according to lasso ranking alone, performed well with respect to internal prediction (AUROCCs: 77-89\%). A calculator for predicted probability of non-adherence and non-persistence outcomes using the items in Lasso-10, is available online (http://www.med.unc.edu/ncaidstraining/adherence/forproviders), providing a short survey that would be suitable in a clinical setting.

The final IRT prediction models included either the IRT scores or items from HIV-ASES, ACTG-ABQ, Difficulty, and Positivity ( 30 items total). Internal prediction of the observed non-adherence ratio within a threshold of $\pm 10 \%$ was achieved for $37 \%$ of individuals when the 30 -item scale was fit using its four continuous IRT scores, and for $47 \%$ of individuals when fit using the 30 individual items from the IRT-30 scale (Supplementry Fig. S1). Looking at dichotomized outcomes with the selected IRT subscales fit as IRT scores, the AUROCC ranged from 63 to $77 \%$ across the dichotomized outcomes. There was numerically stronger prediction when we fit the 30 chosen individual items (AUROCCs: 84-86\%, as shown in Table 2) compared to fitting these same items using their respective IRT scores. We chose to keep IRT subscales intact within the final IRT-30 survey because these items were calibrated as a set in the IRT analyses. The purpose of IRT is to define and evaluate scales based on sets of items measuring a common construct; selecting items from different subscales and combining them into a new scale changes the construct that is being measured. To preserve the original substantive interpretations of the subscales, we did not omit or shuffle items across the IRT subscales. Among the chosen IRT subscales, ACTG-ABQ (adherence barriers) was most strongly associated with non-adherence ( $p$-values $<.001)$ and non-persistence 
outcomes ( $p$-values $=.02$ ). A higher ACTG-ABQ score was associated with greater odds of both non-adherence and non-persistence (Table 3).

\section{Discussion}

Using a comprehensive scale development approach we identified two new scales, both of which were reliable and predictive of medication adherence and persistence among a diverse group of patients living with HIV infection. Our formative work included extensive interviews with service health care providers and focus groups of people living with HIV infection, and set a strong foundation upon which to introduce a new candidate set of items to existing sets from the literature.

The candidate items and previously existing items were combined in a survey and measured in a cohort of patients receiving HIV care and, in general, taking HIV therapy for several years. Among these participants we found that adherence to ART was suboptimal approximately one quarter of the time, with $27 \%$ of participants missing doses more than $30 \%$ of the days they were observed. Further, $17 \%$ had at least a week-long break in therapy during study follow-up.

For prediction of suboptimal adherence and non-persistence outcomes we identified a new ten-item scale using the lasso variable selection method. Eight of the top ten items for prediction of adherence and persistence were novel to this study. This ten-item scale had marginal reliability of.77, which is reasonable reliability for a scale of this length, and had area under the ROC curve internal prediction values of 84.6 and $88.6 \%$ for $>30 \%$ nonadherence and 7-day non-persistence, respectively. This new scale takes approximately 5 min to complete and has potential for future evaluation and clinical use.

When examining the ability of items derived from our formative work and from the literature to predict lapses in therapy, we found that those assessing perceived confidence in medication taking and self-reported barriers to adherence were strongest in independently predicting non-adherence. Items related to barriers to adherence (e.g., affordability, integration with schedule and activities) were best able to predict independently higher levels of non-adherence, as well as 3- and 7-day non-persistence. Self-reported adherence measured using the visual analog scale [[15] ] at study entry did not improve the predictive capability of the selected multivariable models.

Unlike many scale development studies, we further applied an in-depth IRT psychometric approach to identify new item subscales that were both short and reliable. Using this approach, we identified new IRT subscales and ultimately a 30-item scale with high marginal reliability (.90) and internal predictive capability (as measured by area under the ROC curve) similar to our ten-item scale. This 30-item scale includes two previously existing subscales plus two novel subscales and takes approximately $10 \mathrm{~min}$ to complete. Arriving at and interpreting the psychologically meaningful construct is the IRT goal, while purely predicting medication adherence and persistence outcomes is the goal of the lasso method. Each approach contributes unique information, and one would not necessarily expect the two approaches to converge on the same sets of items. When interpreted side by side, these two methods can help identify which sets of items measure a common psychological construct, and which individual items are the strongest predictors of adherence.

Interestingly, the IRT subscales derived from the formative work were short and reliable, but were weaker at predicting adherence and persistence as those previously developed in other populations [[13] , [14] ]. This finding may reflect a disconnect between what people living with HIV report as being facilitators and barriers to adherence and what actually influences medication taking. The longest of our new IRT subscales had only six items, compared to 9 and 12 items in the ACTG-ABQ and HIV-ASES subscales, respectively, also suggesting that our formative work produced new items that spanned across several psychological constructs. It should also be noted that the IRT scales were formed not based on their ability to predict adherence, but rather on the degree 
to which responses on individual items co-vary to measure a common psychological construct. While a set of items measuring disparate constructs may predict medication adherence well, these items do not necessarily produce a unidimensional, reliable IRT subscale. To be considered an IRT subscale, it is assumed that the same underlying psychological construct influences the item responses within the set. Likewise, the item set most predictive of adherence (i.e., the ten-item scale identified using the lasso approach) is not necessarily a set of items that forms a psychologically meaningful construct.

A major strength of this investigation was the economic, educational, racial/ethnic, and gender diversity of our studied sample. In addition, this contemporary cohort taking HIV medications reflects the characteristics of patients of the foreseeable future; most were on once-daily therapy. This study had high retention rates, and there were relatively little missing data. Our candidate items were developed via extensive formative research conducted with individuals not unlike those studied. In addition, items from select instruments that had been previously reported to be predictive were included in the survey battery. As there is some variability in the methods used to estimate adherence using MEMs, we opted to conduct analyses using two approaches: a strict conservative use of the MEMs cap openings such that only the MEMs data were evaluated, and an adjudicated use that included 'forgiveness' for explained non-openings of the cap. We found the difference between the two approaches to be minimal-a finding that can provide guidance to future use and analysis of MEMs data.

Aspects of the study that were strengths were also limitations. The MEMS indices do not tell specifically that medication was actually ingested but rather that a particular medication bottle cap was opened. Other study limitations include the fact that we cannot say which items would perform best with an external set of data, as we used the complete dataset to identify the predictive items and sets. While our study sample size was relatively large, this investigation would benefit from an external validation of the final ten-item and 30-item inventories. Follow-up, on study HIV RNA data were not uniformly available and therefore could not be described. Finally, we tracked study participants for 3 months; longer term patterns of medication adherence and persistence are worthy of additional study.

In summary, as HIV therapy continues to require daily self-administration of one or more oral medications, adherence and persistence to these therapies continues to be essential to treatment success and long term wellbeing. We identified a series of questions that could be used to elicit responses that would be predictive of future ART adherence and persistence including a ten-item battery of items most predictive of these outcomes, as well as a 30-item battery reflective of the psychological constructs found to be the root of medication-taking in this population. These batteries may be useful to clinicians and researchers, respectively, and others seeking to predict ART taking behaviors. The ability to identify individuals at risk for suboptimal adherence can be highly valuable in directing counseling and other supportive measures to achieve and maintain control of HIV.

\section{Electronic supplementary material}

The online version of this article (doi:10.1007/s10461-017-1772-z) contains supplementary material, which is available to authorized users.

\section{Acknowledgements}

This study was supported by a research Grant from Merck \& Co., Inc, and the University of North Carolina at Chapel Hill Center for AIDS Research (CFAR), an NIH funded program P30 AI50410. We are grateful to the many service providers and the staff at participating clinics who contributed and the many patients living with HIV infection who generously participated. We thank our dedicated ESTEEM research assistants: Madison Hayes, Katherine Stein, Joseph Murphy, Jazmin Brown, Shawn Jones, and Jasmine Stringer. 


\section{Compliance with Ethical Standards}

\section{Conflict of interest}

The authors have no relevant conflicts of interest to report.

\section{Ethical Approval}

All procedures performed in studies involving human participants were in accordance with the ethical standards of the institutional and/or national research committee and with the 1964 Helsinki declaration and its later amendments or comparable ethical standards.

\section{Informed Consent}

Informed consent was obtained from all individual participants included in the study.

\section{References}

\section{Citations}

1 Thompson MA, Mugavero MJ, Amico KR, Guidelines for improving entry into and retention in care and antiretroviral adherence for persons with HIV: evidence-based recommendations from an international association of physicians in AIDS care panel, Ann Intern Med, 2012, 156, 11, 817, 833, 10.7326/00034819-156-11-201206050-004194044043

2 Mugavero MJ, Davila JA, Nevin CR, Giordano TP, From access to engagement: measuring retention in outpatient HIV clinical care, AIDS Patient Care STDS., 2010, 24, 10, 607, 613, 10.1089/apc.2010.00862965698

3 Rudy BJ, Murphy DA, Harris DR, Muenz L, Ellen J, Patient-related risks for nonadherence to antiretroviral therapy among HIV-infected youth in the United States: a study of prevalence and interactions, AIDS Patient Care STDS., 2009, 23, 3, 185, 194, 10.1089/apc.2008.01622856493

4 Nachega JB, Parienti JJ, Uthman OA, Lower pill burden and once-daily antiretroviral treatment regimens for HIV infection: a meta-analysis of randomized controlled trials, Clin Infect Dis, 2014, 58, 9, 1297, 1307, 10.1093/cid/ciu0463982838

5 Schneider J, Kaplan SH, Greenfield S, Li W, Wilson IB, Better physician-patient relationships are associated with higher reported adherence to antiretroviral therapy in patients with HIV infection, J Gen Intern Med, 2004, 19, 11, 1096, 1103, 10.1111/j.1525-1497.2004.30418.x1494791

6 Halkitis PN, Shrem MT, Zade DD, Wilton L, The physical, emotional and interpersonal impact of HAART: exploring the realities of HIV seropositive individuals on combination therapy, J Health Psychol, 2005, 10, 3, 345, 358, 10.1177/1359105305051421

7 Stirratt MJ, Remien $\mathrm{RH}$, Smith A, The role of HIV serostatus disclosure in antiretroviral medication adherence, AIDS Behav, 2006, 10, 5, 483, 493, 10.1007/s10461-006-9106-6

8 Carr RL, Gramling LF, Stigma: a health barrier for women with HIV/AIDS, J Assoc Nurses AIDS Care, 2004, 15, 5, 30, 39, 10.1177/1055329003261981

9 Ryscavage P, Anderson EJ, Sutton SH, Reddy S, Taiwo B, Clinical outcomes of adolescents and young adults in adult HIV care, J Acquir Immune Defic Syndr, 2011, 58, 2, 193, 197, 10.1097/QAI.0B013E31822D7564

10 Miller LG, Liu H, Hays RD, How well do clinicians estimate patients' adherence to combination antiretroviral therapy?, J Gen Intern Med, 2002, 17, 1, 1, 11, 10.1046/j.1525-1497.2002.09004.x1494993

11 Muessig KE, Panter AT, Mouw MS, Medication-taking practices of patients on antiretroviral hiv therapy: control, power, and intentionality, AIDS Patient Care STDS., 2015, 29, 11, 606, 616, 10.1089/apc.2015.00584638213 
12 Wohl D, Jones S, Brown J, Panter AT, Maiese E. Barriers to medication adherence remain in the era of potent HIV therapy. Abstract 143. 8th International Conference on HIV treatment and prevention adherence. June 2-4, 2013, Miami, Florida.

13 Johnson MO, Neilands TB, Dilworth SE, Morin SF, Remien RH, Chesney MA, The role of self-efficacy in HIV treatment adherence: validation of the HIV treatment adherence self-efficacy scale (HIV-ASES), J Behav Med, 2007, 30, 5, 359, 370, 10.1007/s10865-007-9118-32423379

14 Chesney MA, Ickovics JR, Chambers DB, Self-reported adherence to antiretroviral medications among participants in HIV clinical trials: the AACTG adherence instruments, AIDS Care, 2000, 12, 3, 255, 266, 10.1080/09540120050042891

15 Giordano T, Guzman D, Clark R, Charlebois E, Bangsberg D, Measuring adherence to antiretroviral therapy in a diverse population using a visual analogue scale, HIV Clin Trials, 2004, 5, 2, 74, 79, 10.1310/JFXH-G3X2EYM6-D6UG

16 Cai L, Thissen D, Toit SHC, IRTPRO for Windows [Computer software], 2011, Lincolnwood, Scientific Software International

17 Friedman J, Hastie T, Tibshirani R, Regularization paths for generalized linear models via coordinate descent, J Stat Softw, 2010, 33, 1, 1, 22, 10.18637/jss.v033.i012929880

Table 1. Characteristics of study participant $(n=173)$

\begin{tabular}{|c|c|}
\hline Characteristic & Statistics \\
\hline \multicolumn{2}{|l|}{ Age (years) } \\
\hline Median (Q1, Q3) & $48(39,53)$ \\
\hline Mean (SD) & $46(11)$ \\
\hline Min-max & $21-74$ \\
\hline \multicolumn{2}{|l|}{ Gender } \\
\hline Male & $108(63 \%)$ \\
\hline Female & $61(35 \%)$ \\
\hline Transgender & $3(2 \%)$ \\
\hline \multicolumn{2}{|c|}{ Race/ethnicity (check all that apply) } \\
\hline American Indian & $5(3 \%)$ \\
\hline Black & $140(81 \%)$ \\
\hline Hispanic & $3(2 \%)$ \\
\hline White & $28(16 \%)$ \\
\hline More than 1 race & $6(3 \%)$ \\
\hline \multicolumn{2}{|l|}{ Education level } \\
\hline Less than high school & $43(25 \%)$ \\
\hline High school & $59(34 \%)$ \\
\hline College or beyond & $70(41 \%)$ \\
\hline \multicolumn{2}{|c|}{ Insurance status (check all that apply) } \\
\hline Unemployed/disabled & $104(60 \%)$ \\
\hline Insured for clinic visits & $148(86 \%)$ \\
\hline Insured for medication & $155(92 \%)$ \\
\hline Private health & $11(6 \%)$ \\
\hline Medicare & $43(25 \%)$ \\
\hline Medicaid & $83(48 \%)$ \\
\hline ADAP & $56(33 \%)$ \\
\hline State-sponsored & $9(5 \%)$ \\
\hline Other government plan & $12(7 \%)$ \\
\hline Other plan & $24(14 \%)$ \\
\hline Years since HIV?diagnosis & \\
\hline
\end{tabular}




\begin{tabular}{|l|l|}
\hline Median (Q1, Q3) & $12.6(4.6,18.5)$ \\
\hline Min-max & $0.0-30.4$ \\
\hline Ever take HIV medications & $168(97 \%)$ \\
\hline Number of ART medications & $2(1,3)$ \\
\hline Median (Q1, Q3) & $0-5$ \\
\hline Min-Max & \\
\hline Dosing frequency from self-report & $115(68 \%)$ \\
\hline Once daily & $54(32 \%)$ \\
\hline Twice daily & $142(82 \%)$ \\
\hline Dosing frequency from MEMs & $31(18 \%)$ \\
\hline Once daily & $8.2(3.0,15.6)$ \\
\hline Twice daily & $0.0-28.3$ \\
\hline Years since ART start & $82(48 \%)$ \\
\hline Median (Q1, Q3) & $70(41 \%)$ \\
\hline Min-max & $20(12 \%)$ \\
\hline Type of pharmacy (check all that apply) & $9(5 \%)$ \\
\hline Mail pharmacy & \\
\hline Local pharmacy & $565(350,770)$ \\
\hline Clinic pharmacy & $605(334)$ \\
\hline Other pharmacy (e.g. home delivery) & $10-2097$ \\
\hline CD4 count (cells/mm3) & $132(79 \%)$ \\
\hline Median (Q1,Q3) & $100(96,100)$ \\
\hline Mean (SD) & $96(12)$ \\
\hline Min-max & $0-100$ \\
\hline HIV-1 RNA 50 copies/mL & $103(61 \%)$ \\
\hline Adherence visual analog scale, past 30 days & $79(46 \%)$ \\
\hline Median (Q1, Q3) & \\
\hline Mean (SD) & \\
\hline Min-max & \\
\hline Reported 100\% on adherence visual analog scale & \\
\hline Depression/bipolar diagnosis & \\
\hline Paridant charactics & \\
\hline
\end{tabular}

Participant characteristics were summarized with a complete-record approach (missing data excluded). Missing data: age $(n=4)$, gender, education level, insurance status, number of ART medications, type of pharmacy $(n=1$ each), years since HIV diagnosis $(n=6)$, years since ART start $(n=23)$, CD4 cell count $(n=9), \operatorname{HIV}-1$ RNA $(n=5)$, and adherence visual analog scale $(n=4)$

Table 2 Prediction of non-adherence and non-persistence

\begin{tabular}{|l|c|c|c|c|c|}
\hline Endpoint & Events/total & $\begin{array}{l}\text { AUROCC IRT } \\
\text { scores }^{\mathrm{a}}\end{array}$ & Events/total & $\begin{array}{l}\text { AUROCC IRT } \\
30^{\mathrm{b}, \mathrm{c}}\end{array}$ & $\begin{array}{l}\text { AUROCC Lasso } \\
10^{\mathrm{c}, \mathrm{d}}\end{array}$ \\
\hline $\begin{array}{l}\text { Non-adherence } \\
>20 \%\end{array}$ & $66 / 169$ & .700 & $68 / 173$ & .861 & .813 \\
\hline $\begin{array}{l}\text { Non-adherence } \\
>30 \%\end{array}$ & $45 / 169$ & .766 & $47 / 173$ & .841 & .846 \\
\hline $\begin{array}{l}\text { Non-persistence 3- } \\
\text { day }\end{array}$ & $62 / 169$ & .633 & $64 / 173$ & .838 & .766 \\
\hline $\begin{array}{l}\text { Non-persistence 7- } \\
\text { day }\end{array}$ & $28 / 169$ & .696 & $29 / 173$ & .835 & .886 \\
\hline & & $4 \mathrm{df}$ & & $30 \mathrm{df}$ & $13 \mathrm{df}$ \\
\hline
\end{tabular}

HIV-ASES the HIV treatment adherence self-efficacy scale, ACTG-ABQ modified version of the AIDS clinical trials group adherence barriers questionnaire, Difficulty, Positivity additional ESTEEM items created based on stakeholder and consumer interviews, AUROCC area under receiver-operator characteristic curve, df degrees of freedom, IRT item response theory, Lasso least absolute shrinkage and selection operator a HIV-ASES, ACTG-ABQ, Difficulty and Positivity fit as 4 continuous IRT scores; $n=169$ ( 4 subjects with missing IRT scores excluded) 
b HIV-ASES, ACTG-ABQ, Difficulty and Positivity fit using the 30 Likert-type survey items included in these IRT subscales $\mathrm{c} n=173$, average AUROCC over ten imputations is shown

$\mathrm{d}$ Top ten items using lasso selection (includes three contingency items with three categories each, hence $13 \mathrm{df}$ )

Table 3 Association between IRT Scores and adherence and persistence $(n=169)$

\begin{tabular}{|c|c|c|c|c|c|}
\hline & Rate ratio & $95 \% \mathrm{Cl}$ & & Wald Chi square & P value \\
\hline \multicolumn{6}{|c|}{ Non-adherence rate } \\
\hline HIV-ASES & 1.25 & 1.01 & 1.53 & 4.38 & .04 \\
\hline ACTG-ABQ & 1.61 & 1.25 & 2.07 & 13.51 & $<.001$ \\
\hline Difficulty & 1.08 & 0.86 & 1.35 & 0.40 & .53 \\
\hline \multirow[t]{2}{*}{ Positivity } & 0.86 & 0.69 & 1.08 & 1.66 & .20 \\
\hline & Odds ratio & $95 \% \mathrm{Cl}$ & & Wald Chi square & $P$ value \\
\hline \multicolumn{6}{|c|}{ Non-adherence $>20 \%$} \\
\hline HIV-ASES & 1.11 & 0.76 & 1.61 & 0.29 & .59 \\
\hline ACTG-ABQ & 2.18 & 1.38 & 3.43 & 11.30 & $<.001$ \\
\hline Difficulty & 1.22 & 0.78 & 1.89 & 0.76 & .38 \\
\hline Positivity & 0.70 & 0.45 & 1.09 & 2.49 & .11 \\
\hline \multicolumn{6}{|c|}{ Non-adherence $>30 \%$} \\
\hline HIV-ASES & 1.44 & 0.93 & 2.25 & 2.65 & .10 \\
\hline ACTG-ABQ & 2.79 & 1.71 & 4.57 & 16.70 & $<.001$ \\
\hline Difficulty & 1.37 & 0.82 & 2.28 & 1.46 & .23 \\
\hline Positivity & 0.71 & 0.42 & 1.21 & 1.58 & .21 \\
\hline \multicolumn{6}{|c|}{3 day non-persistence } \\
\hline HIV-ASES & 0.88 & 0.62 & 1.27 & 0.45 & .50 \\
\hline ACTG-ABQ & 1.67 & 1.08 & 2.58 & 5.27 & .02 \\
\hline Difficulty & 1.31 & 0.85 & 2.04 & 1.48 & .22 \\
\hline Positivity & 0.97 & 0.63 & 1.50 & 0.02 & .89 \\
\hline \multicolumn{6}{|c|}{7 day non-persistence } \\
\hline HIV-ASES & 1.25 & 0.77 & 2.03 & 0.81 & .37 \\
\hline ACTG-ABQ & 1.87 & 1.11 & 3.13 & 5.55 & .02 \\
\hline Difficulty & 1.49 & 0.83 & 2.68 & 1.81 & .18 \\
\hline Positivity & 1.19 & 0.67 & 2.14 & 0.35 & .55 \\
\hline
\end{tabular}

Bold values indicate statistical significance HIV-ASES the HIV treatment adherence self-efficacy scale, ACTG-ABQ modified version of the AIDS clinical trials group adherence barriers questionnaire, Difficulty, Positivity additional ESTEEM items created based on stakeholder and consumer interviews a Multivariable models were fit using continuous IRT scores for HIV-ASES, ACTG-ABQ, Difficulty and Positivity. A non-adherence rate ratio was estimated using negative binomial regression (dispersion estimate $=1.33,95 \% \mathrm{Cl} 1.07-1.65$ ), and odds ratios were estimated using logistic regression

Table 4 Item content, number of items and marginal reliabilities for 12 item response theory (IRT) subscales

\begin{tabular}{|c|c|c|}
\hline IRT subscale name and items & $\begin{array}{l}\text { Total } \\
\text { items }\end{array}$ & $\begin{array}{l}\text { IRT } \\
\text { marginal } \\
\text { reliability }\end{array}$ \\
\hline HIV-ASES/confidence: In the next 30 days, how confident are you that you can: & 12 & .75 \\
\hline
\end{tabular}




\begin{tabular}{|c|c|c|}
\hline $\begin{array}{l}\text { 1. Stick to taking your HIV medicines even when side effects begin to interfere with daily } \\
\text { activities? } \\
\text { 2. Integrate taking your HIV medicines into your daily routine? } \\
\text { 3. Integrate taking your HIV medicines into your daily routine even if it means taking them } \\
\text { around other people who don't know you are HIV-infected? } \\
\text { 4. Stick to your HIV medicine schedule even when your daily routine is disrupted? } \\
\text { 5. Stick to your HIV medicine schedule when you aren't feeling well? } \\
\text { 6. Stick to your HIV medicine schedule when it means changing your eating habits? } \\
\text { 7. Continue with taking your HIV medicines even if doing so interferes with your daily activities? } \\
\text { 8. Continue with the HIV medicines plan your physician prescribed even if your T-cells drop } \\
\text { significantly in } \\
\text { the next } 3 \text { months? } \\
\text { 9. Continue with the HIV medicines even when you are feeling discouraged about your health? } \\
\text { 10. Continue with taking your HIV medicines even when getting to your clinic appointments is a } \\
\text { major hassle? } \\
\text { 11. Continue with taking your HIV medicines even when people close to you tell you that they } \\
\text { don't think that it is doing any good? } \\
\text { 12. Continue taking your HIV medicines even if it doesn't make you feel better? }\end{array}$ & & \\
\hline Social support & 4 & .83 \\
\hline $\begin{array}{l}\text { 1. I have some very close people in my life who would do almost anything for me } \\
\text { 2. My support (friends, family) give me strong emotional support } \\
\text { 3. I have friends who give me strong emotional support } \\
\text { 4. I have family who give me strong emotional support }\end{array}$ & & \\
\hline Well-informed/doctor-patient relationship & 5 & .75 \\
\hline $\begin{array}{l}\text { 1. It makes me feel strong when I get a positive health report from my doctor } \\
\text { 2. I would tell the doctor if I weren't taking my medication } \\
\text { 3. I have come to accept my HIV diagnosis } \\
\text { 4. I have learned a great deal from reading about HIV/AIDS } \\
\text { 5. I am comfortable asking my doctor questions about my disease }\end{array}$ & & \\
\hline Difficulty/interference of medication & 5 & .83 \\
\hline $\begin{array}{l}\text { 1. I have physical health problems that make it hard for me to take my HIV meds regularly } \\
\text { 2. I have mental health problems that make it hard for me to take my HIV meds regularly } \\
\text { 3. It is hard for me to keep track of my HIV meds } \\
\text { 5. HIV medications interfere with my ability to have fun }\end{array}$ & & \\
\hline Positivity about medication & 4 & .80 \\
\hline $\begin{array}{l}\text { 1. I feel pretty healthy when I take my HIV medications } \\
\text { 2. When I take my HIV medications, I feel better about myself } \\
\text { 3. Taking my HIV medications gives me hope } \\
\text { 4. Taking HIV medication reminds me to take care of my personal health }\end{array}$ & & \\
\hline Management of medication & 3 & .82 \\
\hline $\begin{array}{l}\text { 1. I'd take my HIV meds more consistently if my doctor(s) helped me figure out if I was actually } \\
\text { ready to take the HIV meds } \\
\text { 2. I'd take my HIV meds more consistently if my doctor(s) told me more about the problems I } \\
\text { might face with getting my HIV pills (such as cost) } \\
\text { 3. I'd take my HIV meds more consistently if my doctor(s) helped me balance taking my HIV } \\
\text { meds with my other health needs (e.g., heart, weight, mental health, substance abuse) }\end{array}$ & & \\
\hline Effects of medication & 3 & .84 \\
\hline $\begin{array}{l}\text { 1. I'd take my HIV meds more consistently if my doctor(s) taught me how the HIV meds help me } \\
\text { stay healthy } \\
\text { 2. I'd take my HIV meds more consistently if my doctor(s) showed me how the HIV meds affect } \\
\text { my lab/blood results } \\
\text { 3. I'd take my HIV meds more consistently if my doctor(s) were very clear with me about the } \\
\text { side effects of my HIV medication }\end{array}$ & & \\
\hline Trust: do you trust your health care provider: & 4 & .55 \\
\hline 1. To offer you high quality medical care? & & \\
\hline
\end{tabular}




\begin{tabular}{|c|c|c|}
\hline $\begin{array}{l}\text { 2. To be more concerned about your health than the time, effort, and costs of treating you? } \\
\text { 3. To prescribe the best HIV medications? }\end{array}$ & & \\
\hline $\begin{array}{l}\text { ACTG-ABQ: In the past month, have you missed taking your medications because you: Please check } \\
\text { one box } \\
\text { for each question. }\end{array}$ & 9 & .90 \\
\hline $\begin{array}{l}\text { 1. Forgot? } \\
\text { 2. Didn't get prescription; ran out of pills? } \\
\text { 3. Busy doing other things (e.g., working, trying to survive, getting food)? } \\
\text { 4. Having to wake up very early to go to work and no time to eat? } \\
\text { 5. Was too busy at work, school, or home? } \\
\text { 6. Didn't want to bring my pills to social activities (restaurant, friend's home)? } \\
\text { 7. Wanted to have a free day without pills? } \\
\text { 8. Lost track of time? } \\
\text { 9. Didn't have a good night sleep? }\end{array}$ & & \\
\hline Pleasantness & 3 & .88 \\
\hline $\begin{array}{l}\text { 1. I'd take my HIV meds more consistently if the pills were more pleasant to take } \\
\text { 2. I'd take my HIV meds more consistently if the pills were smaller. } \\
\text { 3. I'd take my HIV meds more consistently if the pills tasted more like candy }\end{array}$ & & \\
\hline Mode of administration & 4 & .76 \\
\hline $\begin{array}{l}\text { 1. I'd take my HIV medications more consistently if I could take the pills in a liquid form rather } \\
\text { than tablets } \\
\text { 2. I'd take my HIV medications more consistently if they were a shot instead of pills } \\
\text { 3. I'd take my HIV medications more consistently if they were a shot taken once a month } \\
\text { 4. I'd take my HIV medications more consistently if I did not have to take them in a special way } \\
\text { (e.g., with } \\
\text { food, on an empty stomach). }\end{array}$ & & \\
\hline Convenience & 6 & .90 \\
\hline $\begin{array}{l}\text { 1. I'd take my HIV meds more consistently if I felt fewer side effects } \\
\text { 2. I'd take my HIV meds more consistently if I had fewer pills to take } \\
\text { 3. I'd take my HIV meds more consistently if the meds were cheaper } \\
\text { 4. I'd take my HIV meds more consistently if they were dosed less frequently } \\
\text { 5. I'd take my HIV meds more consistently if I had been told more about the side effects before } \\
\text { taking the pills in the first place } \\
\text { 6. I had special dispensers to help me keep track of the pills }\end{array}$ & & \\
\hline
\end{tabular}

HIV-ASES the HIV treatment adherence self-efficacy scale, ACTG-ABQ modified version of the AIDS clinical trials group adherence barriers questionnaire

Additional ESTEEM Items created based on stakeholder and consumer interviews. Item subsets were identified using exploratory analyses for ordered categorical variables, measures of item dependence, item trace lines and information functions. Items were rated on a scale ranging from

1 = strongly disagree to $5=$ strongly agree

Table 5 Final ten-item scale

\begin{tabular}{|l|l|l|l|}
\hline Lasso-10 marginal reliability $=.77$ & Response options & $\begin{array}{l}\text { Item } \\
\text { origin }\end{array}$ & $\begin{array}{l}\text { Lasso } \\
\text { prediction } \\
\text { rank }\end{array}$ \\
\hline Items & & & \\
\hline $\begin{array}{l}\text { 1. In the next 30 days, how confident are you that you can continue } \\
\text { with the HIV medicines even when you are feeling discouraged about } \\
\text { your health? }\end{array}$ & $\begin{array}{l}\text { Scale ranging from 0 } \\
\text { ("cannot do at all") to 10 } \\
\text { ("completely certain can } \\
\text { do") }\end{array}$ & ASES & 1 \\
\hline
\end{tabular}




\begin{tabular}{|l|l|l|l|}
\hline $\begin{array}{l}\text { 2. In the past month, have you missed taking your HIV medications } \\
\text { No/yes ABQ because you forgot? }\end{array}$ & No/yes & ABQ & 3 \\
\hline 3. I find it easy to take my HIV meds with the other medication I take & (a) Strongly disagree, & New & 7 \\
\hline $\begin{array}{l}\text { 4. I have reduced my illegal drug use because I am taking my HIV } \\
\text { medications }\end{array}$ & (b) Disagree, & New & 8 \\
\hline & $\begin{array}{l}\text { (c) Neither disagree nor } \\
\text { agree }\end{array}$ & & \\
\hline 5. If I could stop taking illegal drugs, I would be able to take my HIV & (d) Agree, & New & 2 \\
\hline medications regularly & (e) Strongly agree, & & \\
\hline & (f) Does not apply & & \\
\hline 6. People often make me feel badly about being HIV? & (1) Strongly disagree, & New & 4 \\
\hline 7. Taking pills everyday is not a big deal & (2) Disagree, & New & 5 \\
\hline $\begin{array}{l}\text { 8. It is hard for me to keep track of taking my HIV meds } \\
\text { (3) Neither disagree nor } \\
\text { agree, }\end{array}$ & New & 6 \\
\hline 9. Taking my HIV medications gives me hope & (4) Agree, & New & 9 \\
\hline $\begin{array}{l}\text { 10. I can count on my family and friends to make sure I am taking my } \\
\text { HIV meds consistently }\end{array}$ & (5) Strongly agree & New & 10 \\
\hline
\end{tabular}

HIV-ASES the HIV treatment adherence self-efficacy scale. ACTG-ABQ modified version of the AIDS clinical trials group adherence barriers questionnaire. New items created based on stakeholder and consumer interviews in the present study. This ten-item survey has been ordered to group items with similar response scales together. Lasso prediction rank is shown for research purposes, with \#1 being the most predictive item in the present study, as summarized over non-adherence and non-persistence outcomes

Table 6 Final 30-item IRT scale

\begin{tabular}{|l|l|}
\hline IRT-30 scale marginal reliability $=.90$ & $\begin{array}{l}\text { Item } \\
\text { origin }\end{array}$ \\
\hline Items & \\
\hline In the next 30 days, how confident are you that you can: & \\
\hline (11-point scale from 0 [cannot do at all] to 10 [completely certain can do]) & ASES \\
\hline 1. Stick to taking your HIV medicines even when side effects begin to interfere with daily activities? & ASES \\
\hline 2. Integrate taking your HIV medicines into your daily routine? & ASES \\
\hline $\begin{array}{l}\text { 3. Integrate taking your HIV medicines into your daily routine even if it means taking them around other } \\
\text { people who don't know } \\
\text { you are HIV-infected? }\end{array}$ & \\
\hline 4. Stick to your HIV medicine schedule even when your daily routine is disrupted? & ASES \\
\hline 5. Stick to your HIV medicine schedule when you aren't feeling well? & ASES \\
\hline 6. Stick to your HIV medicine schedule when it means changing your eating habits? & ASES \\
\hline 7. Continue with taking your HIV medicines even if doing so interferes with your daily activities? & ASES \\
\hline $\begin{array}{l}\text { 8. Continue with the HIV medicines plan your physician prescribed even if your T-cells drop significantly in } \\
\text { the next 3 months? }\end{array}$ & ASES \\
\hline 9. Continue with the HIV medicines even when you are feeling discouraged about your health? & ASES \\
\hline $\begin{array}{l}\text { 10. Continue with taking your HIV medicines even when getting to your clinic appointments is a major } \\
\text { hassle? }\end{array}$ & ASES \\
\hline $\begin{array}{l}\text { 11. Continue with taking your HIV medicines even when people close to you tell you that they don't think } \\
\text { that it is doing any } \\
\text { good? }\end{array}$ & ASES \\
\hline 12. Continue taking your HIV medicines even if it doesn't make you feel better? & \\
\hline $\begin{array}{l}\text { In the past month, have you missed taking your medications because you: Please check one box for each } \\
\text { question (No/Yes) }\end{array}$ & ASES \\
\hline $\begin{array}{l}\text { 13. Forgot? } \\
\text { 14. Didn't get prescription; ran out of pills? }\end{array}$ & ABQ \\
\hline
\end{tabular}




\begin{tabular}{|l|l|}
\hline 15. Busy doing other things (e.g., working, trying to survive, getting food?)? & $A B Q$ \\
\hline 16. Having to wake up very early to go to work and no time to eat? & ABQ \\
\hline 17. Was too busy at work, school, or home? & ABQ \\
\hline 18. Didn't want to bring my pills to social activities (restaurant, friend's home)? & ABQ \\
\hline 19. Wanted to have a free day without pills? & ABQ \\
\hline 20. Lost track of time? & ABQ \\
\hline 21. Didn't have a good night sleep? & ABQ \\
\hline 5-point Likert scoring for agreement* & \\
\hline 22. I have physical health problems that make it hard for me to take my HIV meds regularly & \\
\hline 23. I have mental health problems that make it hard for me to take my HIV meds regularly & Difficulty \\
\hline 24. It is hard for me to keep track of my HIV meds & Difficulty \\
\hline 25. It's hard for me to take my HIV meds when I am taking other types of medications & Difficulty \\
\hline 26. HIV medications interfere with my ability to have fun & Difficulty \\
\hline 27. I feel pretty healthy when I take my HIV medications & Difficulty \\
\hline 28. When I take my HIV medications, I feel better about myself & Positivity \\
\hline 29. Taking my HIV medications gives me hope & Positivity \\
\hline 30. Taking HIV medication reminds me to take care of my personal health & Positivity \\
\hline
\end{tabular}

HIV-ASES the HIV treatment adherence self-efficacy scale, ACTG-ABQ modified version of the AIDS clinical trials group adherence barriers questionnaire, ESTEEM additional items created based on stakeholder and consumer interviews

* The following items are from our new "Difficulty/Interference of Medication" and "Positivity about Medication" IRT

Subscales

By David A. Wohl; A. T. Panter; Christine Kirby; Brooke E. Magnus; Michael G. Hudgens; Andrew G. Allmon and Katie R. Mollan

AIDS \& Behavior is a copyright of Springer, 2018. All Rights Reserved. 